Received: 2019-10-16

Accepted: 2020-01-21

Published: 2020-05-29

\section{Inadvertent Discogram During LUMBar InTERLaminar EpIdURAL STEROID INJeCTION}

\author{
David S. Stolzenberg, DO' \\ Philip J. Koehler III, DO² \\ Jonathon Teng, MS ${ }^{3}$ \\ Jeremy I. Simon, $\mathrm{MD}^{1}$
}

Background: Interlaminar epidural steroid injections (ILESI) are the most common injection performed for lumbosacral radicular pain. In order to continually improve the performance and safety profile of ILESI, it is imperative to report complications and inadvertent outcomes in addition to studies on efficacy in order to create guidelines to mitigate risk of potential debilitating sequelae.

Case Report: Here we present a case report of a 36-year-old man who underwent a right sided ILESI for right sided lumbosacral radicular pain from a disc herniation. Following the injection, he had complete resolution of right sided symptoms. However, 4 weeks later he developed left sided lumbosacral radicular for which he underwent repeat left sided ILESI that resulted in an inadvertent discogram. Following this procedure a new magnetic resonance image was obtained that revealed a new large left L5-S1 paracentral extrusion with caudal migration of disc material abutting the ligamentum flavum in the path of the left-sided injection attempt. The patient was treated with oral antibiotics and suffered no significant sequelae from the inadvertent discogram.

Conclusions: Discogram during ILESI is a highly unusual and rare complication. We discuss the management and prevention of this complication and review the limited existing literature.

Key words: Anticoagulation guidelines, direct thrombin inhibitors, interventional pain, interventional spine, ischemic pain, neuromodulation

From: 'Department of Physical Medicine and Rehabilitation, Thomas Jefferson University Hospital, Rothman Orthopaedics, Philadelphia, PA; ${ }^{2}$ Department of Rehabilitation, Thomas Jefferson University Hospital, Philadelphia, PA; ${ }^{3}$ Sidney Kimmel Medical College, Thomas Jefferson University, Philadelphia, PA

Disclaimer: There was no external funding in the preparation of this manuscript. Conflict of interest: Each author certifies that he or she, or a member of his or her immediate family, has no commercial association (i.e., consultancies, stock ownership, equity interest, patent/licensing arrangements, etc.) that might pose a conflict of interest in connection with the submitted manuscript. 


\section{BACKGROUND}

Interlaminar epidural steroid injections (ILESI) are the most common intervention performed for lumbosacral radicular pain, followed by the transforaminal (TFESI) and caudal approaches (1). Despite this, ILESI are felt by some to be controversial due to early studies suggesting that they are ineffective, although further review showed these studies to be flawed and of poor quality $(2,3)$. They were eventually followed by multiple doubleblind randomized controlled trials and systematic reviews suggesting strong evidence that ILESI provide good outcomes in the management of lumbosacral radicular pain $(1,4-9)$.

Beyond efficacy, when a clinician is considering any procedure, the risk-to-benefit ratio needs to be carefully evaluated. Epidural steroid injections (ESI) are considered low-risk as supported by 2 large studies with thousands of patients $(10,11)$. An overall complication rate was found to be $2.4 \%$ with all complications classified as minor (10). Albeit rare, severe complications such as paralysis from epidural hematoma on ILESI and spinal cord infarction from TFESI with particulate steroid have been reported (1,10-12). However, the true rate is likely unknown as studies involving complications are mostly limited to case reports $(1,10,12)$.

In order to continually improve the performance and safety profile of ILESI, it is important to report complications and inadvertent outcomes, in addition to studies on efficacy. In this report we discuss a case in which a patient who was successfully treated with an ILESI for lumbosacral radicular pain had a return of symptoms in the contralateral lower limb and underwent repeat ILESI on the contralateral side that resulted in an inadvertent discogram. This is a highly unusual case, as discogram is a rarely reported complication of ESI and it has been much more frequently reported with the transforaminal approach (13-16). It has only been previously reported 3 times in the literature with ILESI, only twice when there was no prior posterior decompressive surgery at the level of injection, and to our knowledge has not been associated with any infectious complications (16-18).

\section{CASE REPORT}

The patient was a 36-year-old man with no significant past medical history who presented to an outpatient physiatric spine specialty clinic with insidious onset of low-back pain and right $\mathrm{S} 1$ distribution radicular pain of 2 months' duration. His pain progressively worsened to the point that it began interfering with his activities of daily living and work. He was trialed on a course of oral corticosteroids and one month of physical therapy with the McKenzie regimen, with minimal pain or functional improvement. Throughout the course, he denied any weakness, bowel or bladder incontinence, saddle anesthesia, or any constitutional symptoms. His only analgesic medication was $800 \mathrm{mg}$ of ibuprofen as needed.

On physical examination, the patient was found to have limited lumbar flexion and a positive right straight leg raise. Muscle strength was graded 5 out of 5 in all bilateral lower-extremity muscle groups. He had decreased sensation to pinprick in the right $\mathrm{S} 1$ distribution along with decreased right Achilles reflex graded 1 out of 4 and no clonus bilaterally. A magnetic resonance imaging (MRI) study from 6 weeks after symptom onset was notable for an L5-S1 central protrusion with annular fissure abutting the right more than the left, traversing the S1 nerve roots. Based on the patient's history, physical examination, and imaging studies, the recommendation was made to proceed with a right interlaminar epidural steroid injection (ILESI).

One week after the initial consultation, an uneventful L5-S1 right paramedian ILESI was performed in routine fashion using fluoroscopic guidance. The patient obtained complete relief of symptoms with restoration of his normal function. However, 3 weeks later, he began noticing recurrent back pain with associated left radicular leg pain in the same classic $\$ 1$ distribution as on the previously painful right side. During the follow-up visit, the physical examination was only notable for a reproduction of left leg pain on left straight leg raise. The decision was therefore made to schedule a left L5-S1 ILESI. A repeat MRI was not obtained, as there was no new trauma or neurological deficits and the prior MRI revealed a mild left lateral recess narrowing from the protrusion bilaterally, albeit right more than left, which could explain the new symptoms.

Under standard sterile conditions, a left-sided L5S1 paramedian ILESI was attempted with a 3.5-inch 20-gauge Touhy needle at the midpoint between the left lamina and spinous process (Fig. 1). During injection, a slight change but not full loss of resistance to air was felt with the needle tip at the ventral interlaminar line (VILL). Under the contralateral oblique (CLO) view, live fluoroscopic imaging demonstrated the spread of contrast medium partially dorsal and superficial to the VILL with a pocket of contained contrast medium located at the line, presumed to be in the ligamentum flavum (Fig. 
2). In the lateral view, the needle was slightly advanced ventrally to approximately the midpoint of the facet joint, with another slight change of resistance felt. Upon additional real-time visualization of the contrast medium, clear intradiscal flow of contrast medium was demonstrated (Fig. 3). The needle was retracted approximately one centimeter behind the VILL, repositioned more medially in the interlaminar space with loss of resistance noted again and contrast medium reinjected, which this time portrayed clear epidural flow of contrast medium. There was, however, also residual contrast medium extending from the VILL directly into the disc in CLO view, indicating that the disc herniation was contiguous with the VILL and ligamentum flavum (Fig. 4). Following negative aspiration, a $5-\mathrm{mL}$ solution containing one $\mathrm{mL}$ of depomedrol $40 \mathrm{mg} / \mathrm{mL}, 1 \mathrm{~mL}$ of $1 \%$ lidocaine, and $3 \mathrm{~mL}$ of saline was administered. Following the procedure, the patient was informed of the intradiscal injection and placed on $500 \mathrm{mg}$ of prophylactic cefazolin three times a day for one week without sequelae. He had complete relief of his symptoms for 2 months with this injection and it was then repeated without event utilizing a midline interlaminar

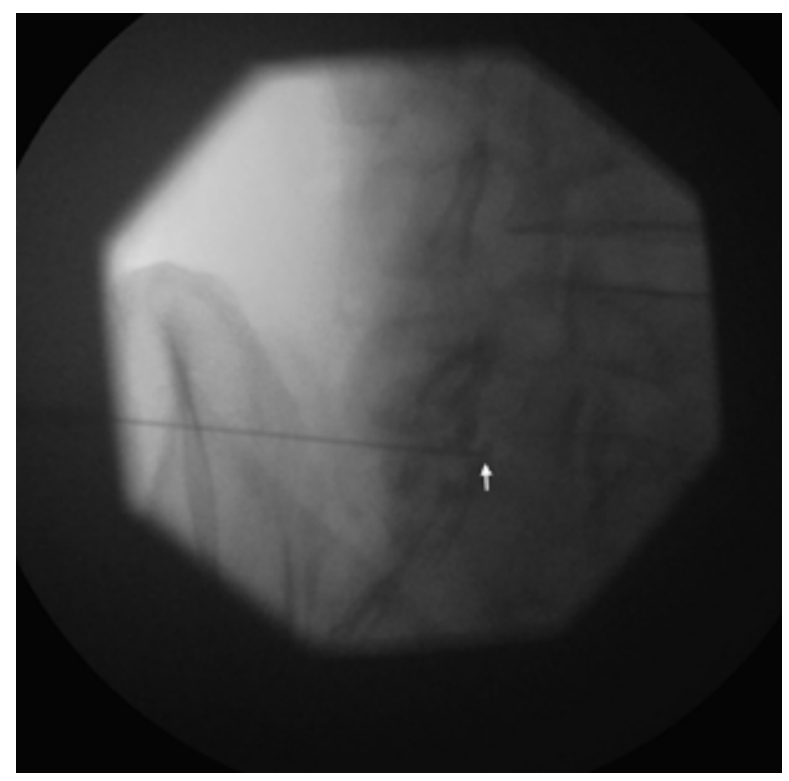

Fig. 2. Contralateral oblique (CLO) fluoroscopic view with needle tip at initial change in resistance at ventral interlaminar line (VILL), demonstrating spread of contrast medium partially dorsal and superficial to the VILL and a pocket of contained contrast medium located at the VILL, presumably in the ligamentum flavum. The white arrow points to the needle tip. approach; however, only minimal short-term relief was obtained and due to persistent severe left-sided S1radicular symptoms, a new MRI was obtained. The new $\mathrm{MRI}$ revealed a large left L5-S1 paracentral extrusion

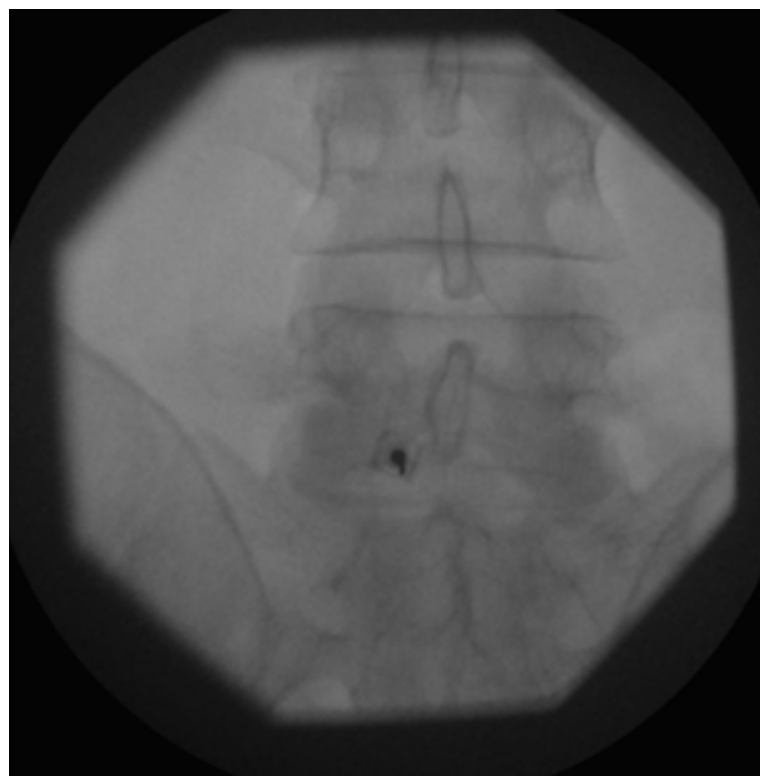

Fig. 1. Anterior-posterior (AP) fluoroscopic trajectory view of the Touhy needle placed for the initial attempt on the left L5-S1 paramedian interlaminar epidural steroid injection (ILESI).

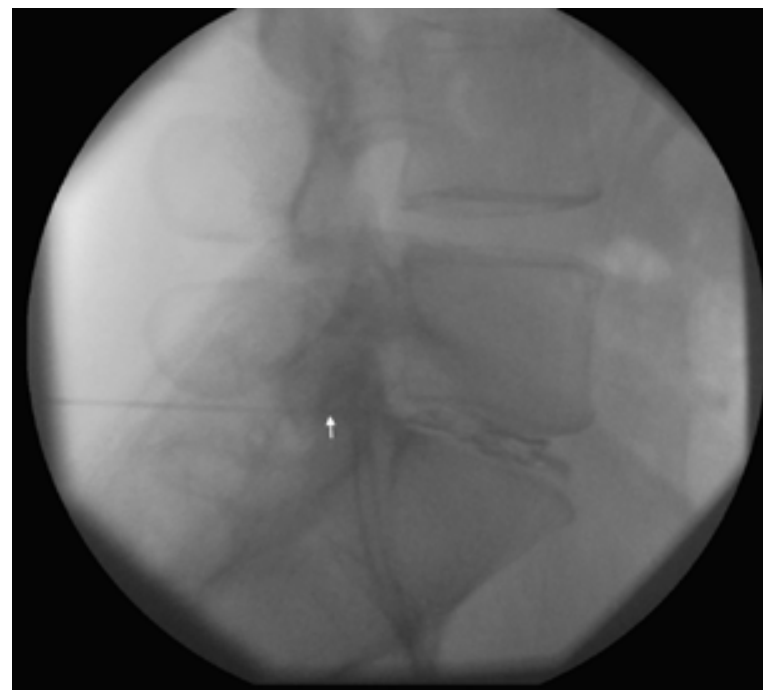

Fig. 3. Lateral view demonstrating inadvertent intradiscal spread of contrast medium after additional needle advancement and loss of resistance. The white arrow points to the needle tip. 


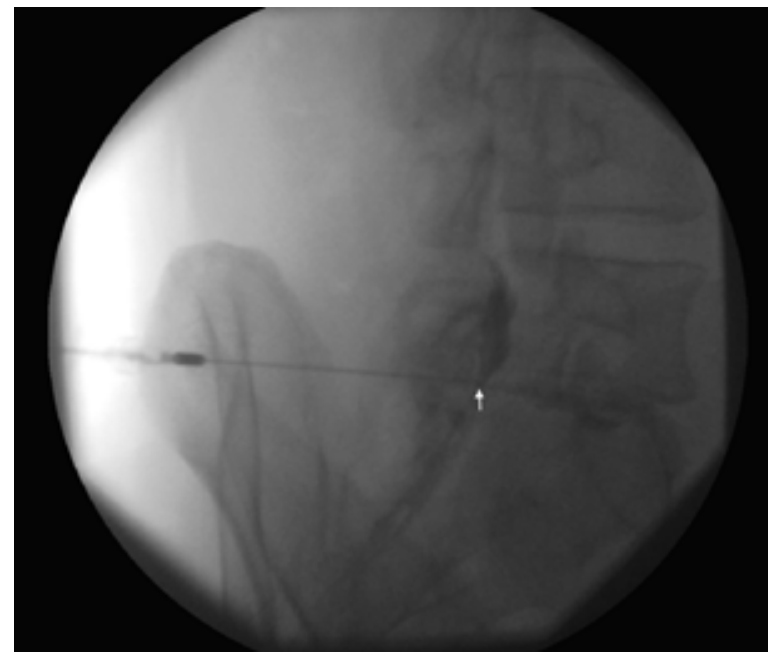

Fig. 4. CLO view demonstrating epidural flow of contrast medium after needle repositioning as well as residual contrast extending from the VILL and into the disc. The white arrow points to the needle tip. with caudal migration of disc material abutting the ligamentum flavum in the path of the initial left-sided injection attempt (Figs. 5-6). At the follow-up appointment, the patient declined the option of additional ESI via the left S1 transforaminal approach or additional physical therapy. He was referred for surgical consultation and underwent a successful L5-S1 microdiscectomy the following month.

\section{DISCUSSION}

ILESI is considered an effective and safe therapeutic injection for lumbosacral radicular pain. A prospective study of 251 patients undergoing TFESI reported a $2.3 \%$ rate of inadvertent disc injection, and a second study suggested a rate of $2.5 \%$ in 2400 injections $(15,16)$. The rate during interlaminar approach is still unknown. In the first reported similar case during ILESI by Huang et al (17), they suggested the needle entered the disc via a triangle composed of the nerve root laterally, dura medially, and pedicle at the base, as the patient did

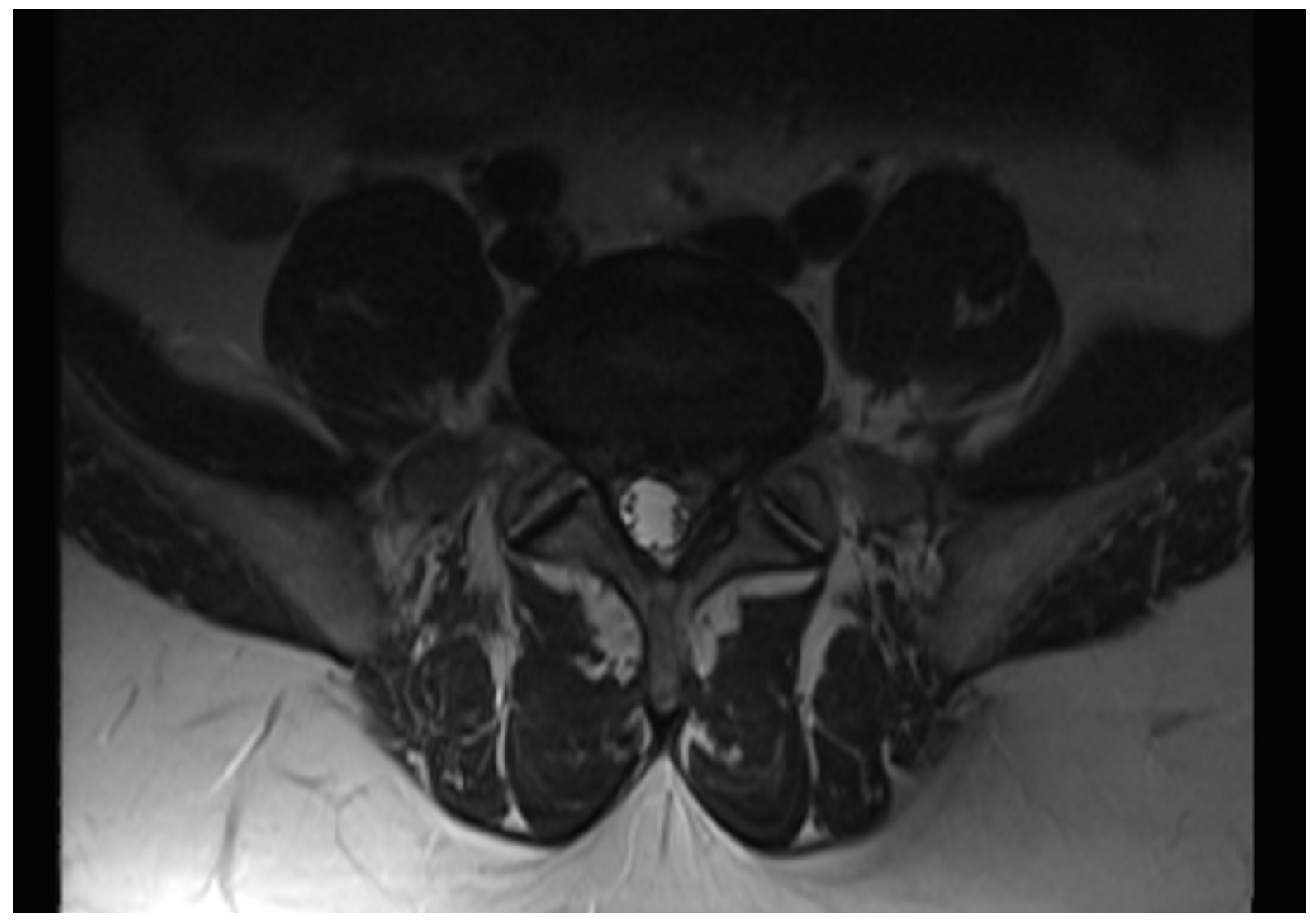

Fig. 5. Updated magnetic resonance imaging (MRI) obtained after the second injection, with axial view at L5-S1 demonstrating left paracentral disc extrusion obliterating the lateral recess. 
not experience a postdural headache or signs of dural puncture. In a second report by Candido et al (16), inadvertent disc injection happened during a similar loss-of-resistance technique with advancement under fluoroscopic visualization; however, this was on a patient that had a prior laminectomy and discectomy at the same level. Posterior decompressive surgery alters the typical spinal anatomy and is generally regarded as a contraindication to interlaminar epidural injections at the same level, so this complication is not completely unexpected. In the third case by Amoretti et al (18), there was the rare complication of sudden onset of paraplegia due to a massive disc fragment that presumably enlarged during intradiscal injection and required emergent decompression. The patient did well following surgery with resolution of the majority of neurologic deficits, but this was the most significant morbidity reported. Therefore, only 2 prior cases of this complication have been reported in a patient without surgery at the injected level. As suggested by all of these reports, it is possible that intradiscal injection is underrecognized and/or underreported, so a true rate is difficult to determine.

Discitis has been rarely reported in case reports of ESI without inadvertent discogram, but it would be assumed that disc entry would increase the risk $(19,20)$. In spite of this, to our knowledge, it has not been reported during inadver-

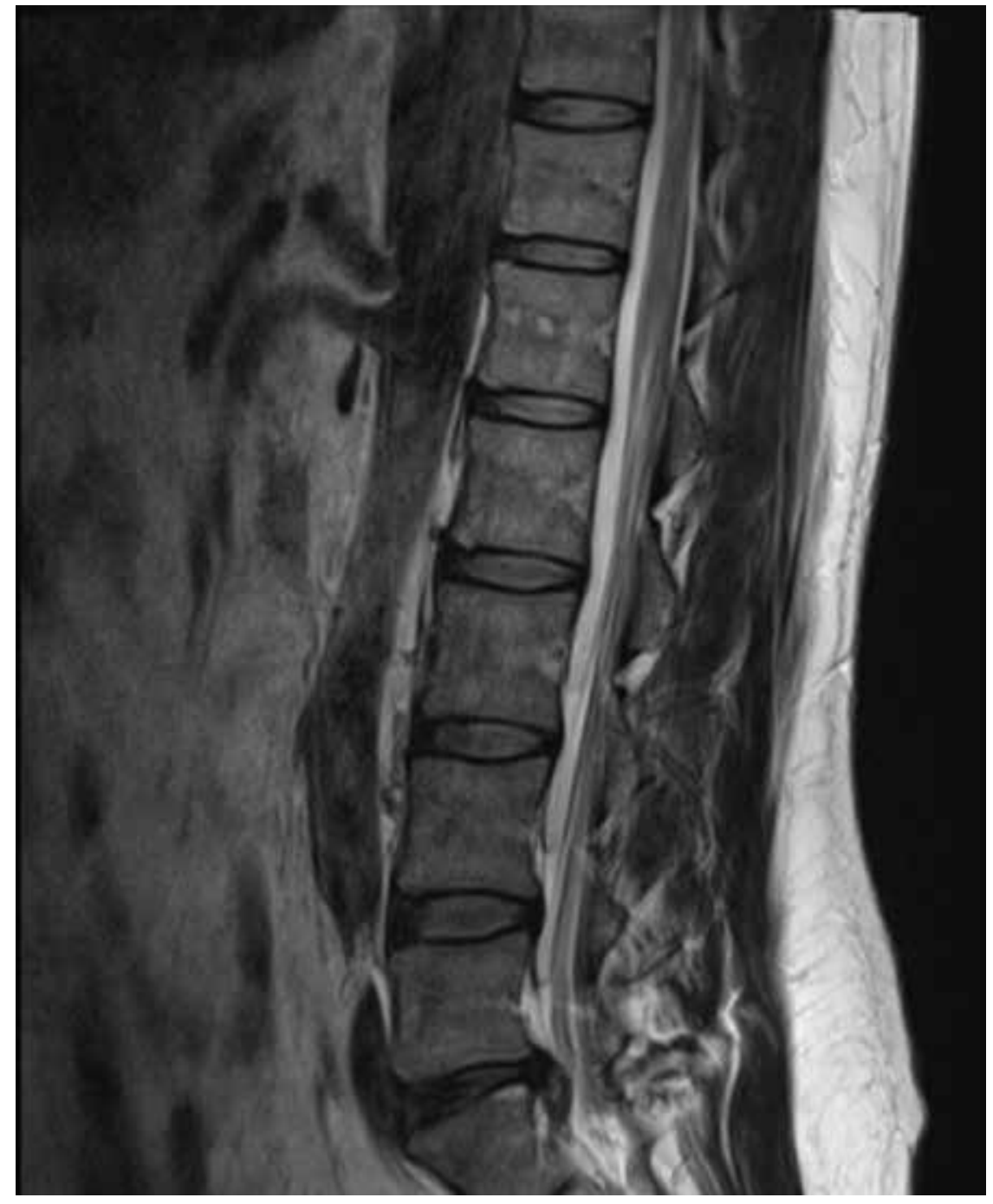

Fig. 6. Left parasagittal view of the MRI with large left L5-S1 paracentral extrusion with caudal migration of disc material.

tent discogram from ESI. There are reported rates of discitis of $0.1 \%$ to $0.3 \%$ per patient and $0.05 \%$ to $0.1 \%$ per disc in discography (21). Therefore, it would not seem unreasonable to infer a higher incidence of infection from inadvertent discogram during ESI versus discography due to the superior aseptic techniques and precautions typically used during discography. Preoperative intravenous antibiotics and/or intradiscal antibiotics, full sterile gown and draping, and double-needle technique are all common for discography. In this report, the patient was treated with oral cephalexin with no signs or symptoms of discitis on follow-up. Two other studies reported using prophylactic antibiotics following inadvertent intradiscal contrast medium flow during ESI. In one report of 6 TFESI cases, the patients were given one gm of intravenous cefazolin following 
evidence of intradiscal contrast medium and had no reports of discitis (15). Another report describes the cases of one ILESI and 4 TFESI patients who were also given $1 \mathrm{~g}$ of intravenous cefazolin and had no reports of discitis (16).

This leads us to recommend oral or intravenous antibiotics whenever there is evidence of inadvertent disruption of disc integrity during an ESI, which has also been suggested by other studies $(15,22)$. One could consider, when feasible, to immediately administer intradiscal antibiotic in the event of inadvertent discogram during ESI. Even further, we wonder whether or not aggressive precautions such as prophylactic perioperative antibiotic administration, similar to surgical patients, should be considered in high-risk or immunocompromised patients before ESI. However, with the rarity of inadvertent intradiscal injection and general lack of immediatelyavailable antibiotic to place intradiscally after ESI, neither of these would seem practical.

We also suggest a lower threshold for repeating an MRI before performing additional injections in patients who experience quantitative increases or change in symptom location. This was also suggested by Cohen et al (14) in their 2008 case report of inadvertent discogram via a transforaminal approach. Interestingly, the same author conducted a multicenter randomized control trial in 2012 that demonstrated that MRI did not change physician decision-making or improve outcomes for patients who had clinical lumbosacral radiculopathy and were referred for ESI (23). The imaging would have changed management in this case. If the MRI was repeated prior to the second injection and demonstrated a new large left paracentral extrusion abutting the ligamentum flavum, this would have prompted a transforaminal approach or a more midline interlaminar entry point. We feel the needle entered the disc immediately as it passed through the ligamentum flavum, and therefore a transforaminal or midline interlaminar approach would have prevented this complication.

There also has to be consideration that the inadvertent discogram caused the new disc herniation seen on repeat MRI. This seems unlikely in this case, as upon needle retraction, the thin tract of contrast medium appeared to clearly extend directly from the ligamentum flavum at the VILL and into the disc space, indicating the 2 structures were contiguous. Herniated discs following discography have been reported in a case series (23) and there has been a prospective cohort study that showed a 2.5-fold increased risk of newdisc herniation after discography compared to controls after 10 years. However, many of the patients in this trial received high-pressure discography, which may have altered the results (24).

Physicians need to be aware of this potential complication and its proper management. Guidelines, however, do not exist to prevent potentially debilitating sequelae such as pyogenic discitis and further reports of complications and research is needed in order to create proper guidance. 


\section{References}

1. Sharma AK, Vorobeychik $Y$, Wasserman $R$, et al. The effectiveness and risks of fluoroscopically guided lumbar interlaminar epidural steroid injections: A systematic review with comprehensive analysis of the published data. Pain Med 2017; 18:239-251.

2. Carette $S$, Leclaire $R$, Marcoux $S$, et al. Epidural corticosteroid injections for sciatica due to herniated nucleus pulposus. N Engl J Med 1997; 336:1634-1640.

3. Pinto RZ, Maher CG, Ferreira ML, et al. Epidural corticosteroid injections in the management of sciatica: A systematic review and meta-analysis. Ann Intern Med 2012; 157:865-877.

4. Benyamin RM, Manchikanti L, Parr AT, et al. The effectiveness of lumbar interlaminar epidural injections in managing chronic low back and lower extremity pain. Pain Physician 2012; 15:363-404.

5. Manchikanti L, Singh V, Cash KA, et al. The role of fluoroscopic interlaminar epidural injections in managing chronic pain of lumbar disc herniation or radiculitis: A randomized, double-blind trial. Pain Pract 2013; 13:547-558.

6. Manchikanti L, Singh V, Cash KA, et al. A randomized, doubleblind, active-control trial of the effectiveness of lumbar interlaminar epidural injections in disc herniation. Pain Physician 2014; 17:61-74.

7. Abdi S, Datta S, Trescot AM, et al. Epidural steroids in the management of chronic pain: A systematic review. Pain Physician 2007, 10:185-212.

8. Chou R, Hashimoto R, Friedly J, et al. Epidural corticosteroid injections for radiculopathy and spinal stenosis: A systematic review and meta-analysis. Ann Intern Med 2015; 163:373-381.

9. Furman MB, Kothari G, Parikh T, et al. Efficacy of fluoroscopically guided, contrast-enhanced lumbosacral interlaminar epidural steroid injections: A pilot study. Pain Med 2010; 11:1328-1334.

10. McGrath JM, Schaefer MP, Malkamaki DM. Incidence and characteristics of complications from epidural steroid injections. Pain Med 2011; 12:726-731.

11. El-Yahchouchi CA, Plastaras CT, Maus TP, et al. Adverse event rates associated with transforaminal and interlaminar epidural steroid injections: A multi-institutional study. Pain Med 2016; 17:239249.

12. Houten JK, Errico TJ. Paraplegia after lumbosacral nerve root block: Report of three cases. Spine J 2002; 2:70-75.

13. Finn KP, Case JL. Disk entry: A complication of transforaminal epi- dural injection-a case report. Arch Phys Med Rehabil 2005; 86:1489-1491.

14. Cohen SP, Maine DN, Shockey SM, et al. Inadvertent disk injection during transforaminal epidural steroid injection: Steps for prevention and management. Pain Med 2008; 9:688-694.

15. Hong JH, Kim SY, Huh B, et al. Analysis of inadvertent intradiscal and intravascular injection during lumbar transforaminal epidural steroid injections: A prospective study. Reg Anesth Pain Med 2013; 38:520-525.

16. Candido KD, Katz JA, Chinthagada, M, et al. Incidence of intradiscal injection during lumbar fluoroscopically guided transforaminal and interlaminar epidural steroid injections. Anesth Analg 2010; 110:1464-1467.

17. Huang J, Kwa A. Lumbar discogram resulting from lumbar interlaminar epidural injection. J Clin Anesth 2004; 16:296-298.

18. Amoretti N, Baqué J, Litrico $S$, et al. Serious neurological complication resulting from inadvertent intradiscal injection during fluoroscopically guided interlaminar epidural steroid injection. Cardiovasc Intervent Radiol 2019; 42:775.

19. Schultz TE. Inadvertent discogram during epidural steroid injection: A case report. AANA J 2008; 76:189-191.

20. Hooten $M$, Mizerak $A, C$ arns $P$, et al. Discitis after lumbar epidural corticosteroid injection: A case report and analysis of the case report literature. Pain Med 2006; 7:46-51.

21. Guyer RD, Ohnmeiss DD. Lumbar discography. Position statement from the North American Spine Society Diagnostic and Therapeutic Committee. Spine 1995; 20:2048-2059.

22. Cohen SP, Gupta A, Strassels SA, et al. Effect of MRI on treatment results or decision making in patients with lumbosacral radiculopathy referred for epidural steroid injections: A multicenter, randomized controlled trial. Arch Intern Med 2012; 172:134-142.

23. Poynton A, Hinman A, Lutz G, et al. Discography-induced acute lumbar disc herniation: A report of five cases. J Spinal Disord Tech 2005; 18:188-192.

24. Carragee EJ, Don AS, Hurwitz EL, et al. 2009 ISSLS Prize Winner: Does discography cause accelerated progression of degeneration changes in the lumbar disc: A ten-year matched cohort study. Spine (Phila Pa 1976) 2009; 34:2338-2345. 
Pain Medicine Case Reports 